\title{
Psychosomatic Molecular Mechanism of Metabolic Syndrome and Type 2 Diabetes. Part 4. A Recovery Strategy from Chronic Hypercapnia and Metabolic Syndrome-Related Disorders, (a Research Proposal)
}

\section{András Sikter MD*}

Internal Medicine, Municipal Clinic of Szentendre, Szentendre, Hungary, Europe

*Corresponding Author: András Sikter MD, Internal Medicine, Municipal Clinic of Szentendre, Szentendre, Hungary, Europe. E-mail: andrassikter3@gmail.com

DOI: 10.31080/ASMS.2020.04.0578
Received: February 17, 2020

Published: February 29, 2020

(C) All rights are reserved by András Sikter.

\begin{abstract}
According to the hypothesis of the author, the Metabolic Syndrome and Type 2 Diabetes are mainly consequences of chronic lowgrade hypercapnia and related intracellular acidosis. The altered $\mathrm{pCO}_{2}$ status changes the Intracellular Ion-Pattern and, consequently, the metabolism. The author has developed a recovery strategy based on his metabolic model, experiences, and data of literature. The primary goal is to increase the minute-ventilation to achieve low-grade hypocapnia ( $\sim 35 \mathrm{mmHg}$ on average) and to maintain it in the long run. As a consequence of the decreased $\mathrm{pCO}_{2}$ level - according to the hypothesis - an intracellular alkaline $\mathrm{pH}$ develops and results in increased metabolism, ATP production, and consequently, phosphate, $\mathrm{Mg}^{2+}, \mathrm{K}^{+}$, and $\mathrm{Zn}^{2+}$ influx into the cytosol. Serum Ca ${ }^{2+}$ levels may also decrease due to increased calcium and phosphate uptake into the 'hungry bones.' It is expected that both the carbohydrates and fats will be burned more intensively in mitochondria, which would also mean a decrease in insulin resistance, serum glucose, and lipid levels. The essence of the method is that regular daily administration of monobasic phosphate $\left(\mathrm{H}_{2} \mathrm{PO}_{4}^{-}\right)$salts as fixed acids can increase respiratory minute volume and decrease the $\mathrm{pCO}_{2}$ level. It is advised to give these salts routinely in doses below the Recommended Dietary Allowance. After achieving the desired $\mathrm{pCO}_{2}$ level, it is advocated that an acidotic $\mathrm{H}_{2} \mathrm{PO}_{4}{ }^{-} / \mathrm{HPO}_{4}{ }^{2-} \mathrm{salt}^{-}$mixture be titrated to maintain the low-grade hypocapnia. Preferably, the cations are provided by combinations of $\mathrm{Mg}^{2+}, \mathrm{K}^{+}$, and $\mathrm{Ca}^{2+}$. The amounts of ions to be administered can be determined by monitoring serum electrolytes. According to the hypothesis, this method could be particularly useful in diseases where elevated levels of $\mathrm{pCO}_{2}$ play a pathophysiological role.

The author also proposes an improved method of Sodi-Pallarés; alternatively, it can be an infusion containing the combination of phosphate buffer, $\mathrm{K}^{+}, \mathrm{Mg}^{2+}, \mathrm{Zn}^{2+}$, and (possibly) other 'cytoplasm-builder' nutrients plus glucose and insulin. Theoretically, it could improve the metabolism of the chronically ill or insulin-resistant patients.

Keywords: Healing with Ion-Mixture Compositions; $\mathrm{HPO}_{4}{ }^{2-}$ as the Second Messenger of Insulin; Modified Method of Sodi-Pallarés; Momentary Intracellular Ion-Pattern Signaling
\end{abstract}

\section{Abbreviations}

ATP: Adenosine Triphosphate; CBIs: Cytoplasm Builder Ions $\left(\mathrm{K}^{+}\right.$, $\mathrm{Mg}^{2+}, \mathrm{Zn}^{2+}, \mathrm{HPO}_{4}{ }^{2-}$ and $\mathrm{H}_{2} \mathrm{PO}_{4}^{-}$); COPD: Chronic Obstructive Pulmonray Disease; GLUT4: Glucose Transporter Type 4; $\mathrm{HPO}_{4}^{2-}$ : Dibasic Phosphate Ion; $\mathrm{H}_{2} \mathrm{PO}_{4}^{-}$: Monobasic Phosphate Ion; IMCL: Intramyo- cellular Lipid; MetS: Metabolic Syndrome; OHS: Obesity Hypoventilation Syndrome; OSA: Obstructive Sleep Apnea; PETCO2: Pressure of End-Tidal Carbon Dioxide; Pi: inorganic Phosphate; RDA: Recommneded Dietary Allowance; Se-P: Serum Phosphate; T2D: Type 2 Diabetes 
Psychosomatic Molecular Mechanisms of Metabolic Syndrome and Type 2 Diabetes. Part 4. A Recovery Strategy from Chronic Hypercapnia and Metabolic Syndrome-Related Disorders, (a Research Proposal)

\section{Introduction}

In PubMed, we will find over 700,000 articles if we are searching for 'diabetes' and over 400,000 if we are asking for 'insulin.' Anyone cannot be able to consider all available information. On the other hand, there are also needs interdisciplinary reviews to go ahead. Therefore, there is space for intuitive, inductive, integrative theses, thoughts. Here is one such theory - without the all-available data.

The purpose of developing a good recovery strategy, we need to know the exact pathophysiology of the disease (in this case, of the Metabolic Syndrome and T2D). The CBIs-ATP-Enzymes cytoplasm model provides a good starting point for that (see Part 1.). Catabolism will develop if any damaging factor (stress) harms the body or any Type II ('cytoplasm-builder') nutrients are missing. The increased cytosolic $\mathrm{H}^{+}$concentration (acidosis) seems to be one of the most potent stimuli to trigger cytoplasmic degradation (catabolism, see Part 1., Part 2. and Part 3.). The downward spiral of catabolism stops when the harmful effects cease and (or) counter-regulatory forces unfold, and then the spiral can start (possibly) upward (see Part 1.).

But what are the primary movers? Which one is the first among equals - of cytoplasmic constituents? Insulin is the hormone that can stop catabolism and trigger anabolism (under certain conditions). That is, it has anti-catabolic effects. But what is the signal that starts the spiral upwards? That signal is also a material, a second messenger, which has to increase the formatting of ATPenergy as all life functions use it up! It is generally accepted that the primary effect of insulin is the 'autophosphorylation,' but the question is whether this can work without ATP production? No, phosphorylation also requires ATP energy. The action of insulin is not intermittent but continuous - so the sequences of events are not necessarily decisive: what comes first and what comes later?

The author presented how we can model the metabolism of cytoplasm in a simple way (Part 1). In Part 2., he asked how can the close coexistence cause a Social Defeat, which, in the case of repetitions or persistent, can lead to chronic low-grade hypercapnia and intracellular acidosis. (It was modelized with caged rodents.) In humans, it can manifest in the clinical pictures of OSA, COPD, OHD, and their formes frustes. These changes lead to altered metabolism, in the first instance, to insulin resistance and lipid metabolism disorders, which may be the same as the Metabolic Synd- rome. In Part 3., the author concludes that lipid beta-oxidation can yield enough ATP narrowly in MetS; when this capacity also starts to decrease, the T2D begins. In this sense, T2D would be a decompensated form of the MetS. At this point, even the firing of glucose and lipids together cannot produce enough ATP energy to perform vital functions adequately. Increased appetite further grows food uptake. These and the raised catabolic processes increase nutrient readiness, temporarily increase serum and cytoplasmic levels of essential metabolite products (as fuel), and their availability for oxidative phosphorylation. - Although in the long term, fuel accumulation in the fat of the body further worsens pathophysiologic conditions, and vicious circles begin. The author argues that one of the primary, most critical actions of insulin is an increase in the cytosolic concentration of $\mathrm{HPO}_{4}^{2-}$ in the target organs (cells). It, as a second messenger, enhances glucose trafficking in the cytoplasm simultaneously by GLUT4 and mitochondrial ATP production, renewing the cytoplasm. In Part 4., the author will present evidence of the literature. On the other hand, he will also look at whether the original Intracellular Ion Pattern of the cells in the body can be restored to recover from diseases? Is there recovery from Type 2 Diabetes or Metabolic Syndrome?

\section{Recovery strategies}

\section{Magnesium deficiency in type 2 diabetes}

A considerable number of scientific literature data related to magnesium and diabetes have been gathered for today. It was thought for decades that primarily the magnesium deficiency is responsible for insulin resistance. (MetS, cardiovascular and other diseases were also accurately studied in this topic). 192 patients with metabolic syndrome were compared with an age-matched healthy control group. Serum magnesium levels were low in $66 \%$, while $4,9 \%$ of the control population ( $\mathrm{P}<0.00001$ ) [1]. Among white participants, low serum magnesium level is a robust and independent predictor of T2D incidents. 12128 nondiabetic middle-aged adults were followed for six years [2]. Among 1453 adults, hypomagnesemia was an average of 8.6 times more common in T2D patients than in healthy clients [3]. Most but not all studies observed hypomagnesemia and/or lacking $\mathrm{Mg}$ nutrition in relationship with T2D [4]. By contrast, the intracellular free $\mathrm{Mg}^{2+}$ levels were consistently decreased in T2D patients compared to nondiabetic individuals [4]. Reduced intake, urinary loss of magnesium played a role in it. Insulin is an essential regulator of magnesium metabolism too. The low cytosolic concentration of magnesium takes part

Citation: András Sikter. "Psychosomatic Molecular Mechanisms of Metabolic Syndrome and Type 2 Diabetes. Part 4. A Recovery Strategy from Chronic Hypercapnia and Metabolic Syndrome-Related Disorders, (a Research Proposal)". Acta Scientific Medical Sciences 4.3 (2020): $150-161$.

DOI: $10.31080 /$ ASMS.2020.04.0578

Published: February 29, 2020 
Psychosomatic Molecular Mechanisms of Metabolic Syndrome and Type 2 Diabetes. Part 4. A Recovery Strategy from Chronic Hypercapnia and Metabolic Syndrome-Related Disorders, (a Research Proposal)

in postreceptorial impairment of insulin action and reinforcement of insulin resistance [2]. Some think that magnesium is a second messenger that regulates directly the glucose metabolism being a cofactor of several enzymes. However, magnesium is not the only player, and there is currently no combination of magnesium in which its administration as a therapeutic agent would have been proposed in Type 2 Diabetes. It seems that Mg-deficiency is both a cause and a consequence of insulin resistance, that is why one participant of the vicious circles (see Part 3.).

\section{Intracellular lack of other Cytoplasm Builder Ions (CBIs) and} cytoplasm builder nutrients in Type 2 Diabetes

T2D is a classic catabolic condition (deterioration of the cytoplasm: see Part 3.) that is initially very difficult to recognize because of its 'stealth' nature. Then catabolism becomes more intense, but does not affect all tissues in the same degree; its course varies from person to person (see Part 1. and Part 3.). Muscle, however, is affected among the first. If we look at Golden's lessons (see Part 1. [24]), we recognize that all cytoplasm-builder (Type II) nutrients decrease in unison in the cytoplasm during catabolism. They can be only rebuilt using all cytoplasm builder nutrients together. In other words: even if a single Type II Nutrient is missing, cells will not build up a lower quality of cytoplasm; they will not produce cytoplasm at all. Let's the constituents of cytoplasm, what's in the literature about lack of them?

Some publications address the risk between hypokalaemia, potassium deficiency in T2D. Potassium deficiency per se impairs glucose tolerance. However, there are no extensive studies to investigate the effect of potassium supplementation on insulin resistance. Potassium may have an additional role in it [5]. According to Rowe et al. [6], the degree of potassium deficiency (estimated from the total body ${ }^{40} \mathrm{~K}$ ) correlated with the decrease in insulin response, which also associated with impaired insulin secretion. It is well known that intracellular $\mathrm{K}^{+}$deficiency and $\mathrm{H}^{+}$overweight are closely related.

It is widely accepted that T2D is associated with high zinc excretion in urine - there is a negative $\mathrm{Zn}^{2+}$ balance - and the serum zinc level is often low, this fact supports the existence of catabolism. An exception is when T2D is treated with insulin because insulin retains $\mathrm{Zn}^{2+}$ [7]. That is, the relationship between insulin and $\mathrm{Zn}^{2+}$ is mutual. Some studies found that zinc deficiency can decrease the response to insulin. However, the mechanism of insulin resistance by zinc is yet unclear. Zinc supplementation for T2D patients ele- vated the abnormally low serum $\mathrm{Zn}^{2+}$ levels and improved glycemic control [8]. Zinc plays a definite role in the synthesis, storage, and secretion of insulin. $\mathrm{Zn}^{2+}$ leaves the beta-cell with insulin; in this way, a higher insulin secretion also could cause cellular depletion of $\mathrm{Zn}^{2+}$ [9]. A comprehensive review [10] selected 53 articles in this topic concluded that "zinc deficiency is associated with glucose intolerance and insulin resistance. However, the effectiveness of the intervention with the zinc supplementation is still inconclusive."

According to Håglin's hypothesis, phosphate metabolism may be a key feature of Metabolic Syndrome; she found evidence for her phosphate theory in women. According to her model, in women, phosphate deficiency could be the common cause of obesity, hypertension, and dyslipidemia [11]. Kalaitzidis et al. confirmed the results of her thesis, with the addition that lower serum magnesium levels also play a significant role in the pathophysiology of the Metabolic Syndrome [12]. Hypophosphatemia can also result in insulin resistance [13]. Phosphate metabolism concerning T2D is complicated by hyperparathyroidism, vitamin D metabolism, and many other factors (see below). Phosphate depletion also impairs insulin secretion [14]. Defective insulin secretion was a consequence of the reduced ATP content and increased cytosolic $\mathrm{Ca}^{2+}$ concentration due to phosphate deficiency.

Although thiamine (Vitamin B1) is not a classical Type II nutrient (see Part 1. [24]), its shortage plays a role in both T2D and obesity. It is a coenzyme of many enzymes that are also involved in carbohydrate metabolism. Perhaps it should be reclassified among Type II Nutrients? Since T2D is generally associated with chronic 'overnutrition' (obesity), it is difficult to believe that T2D starts up a protein and energy deficit. Then it evolves into progressive catabolism and the so-called Sarcopenic Obesity (see Part 3.). It seems that chronic energy deficiency is inseparable from cytoplasmic protein deficiency: from Protein-Energy Malnutrition (see Part 1.). Recently was found that decreased serum levels of five essential and five non-essential amino acids were associated with the prevalence and incidence of T2D [15]. There is a debate about whether deficiencies of specific amino acids can lead to insulin resistance in different populations.

Role of phosphates in signaling. Can phosphate be the second messenger of insulin?

There are many things to suggest that insulin directs the metabolism in target cells through phosphates, namely $\mathrm{HPO}_{4}{ }^{2-}$. It has long been known that $\mathrm{Pi}$ is also a cytosolic signaling molecule that 
Psychosomatic Molecular Mechanisms of Metabolic Syndrome and Type 2 Diabetes. Part 4. A Recovery Strategy from Chronic Hypercapnia and Metabolic Syndrome-Related Disorders, (a Research Proposal)

can control the metabolic rate via regulation of mitochondrial oxidative phosphorylation [16]. Petersen et al. hypothesized that skeletal muscle lipid accumulation (IMCL) is also a consequence of the defects in insulin-dependent phosphate transport (see Part 3. [3]). The increase of cytosolic Pi concentration, as well as decreased in $\mathrm{H}^{+}$concentration, equally play a role in the insulin effect. The cytosolic alkalinizing effect of insulin enhances not only the oxidative phosphorylation [16] but also the cellular glucose traffick via GLUT4 [17].

Some studies suggest that one of the first effects of insulin includes Pi transport; in addition to autophosphorylation [18], and cytosolic alkalization [19]. Before the discovery of insulin, in the 1920s, there were many articles on how can reduce glucose level or even improve the course of diabetes with phosphate therapy, which researches stopped subsided after the discovery of insulin (Part 1. [35]). Then, it was shown that insulin promotes the delivery of Pi to cells [19]. On the other hand, it has also become apparent that without the cytosol alkalinization by insulin, the function of GLUT4 (intracellular trafficking of glucose) and mitochondrial oxidative phosphorylation is also impaired.

According to Laurent Beck (personal communication), who is one of the leading experts of phosphate transporters, there was significant progress in Pi research over the last years. Still, there are also deficiencies, especially in the early signaling of phosphates. Specifical PI3K receptor inhibitors hindered the insulin-stimulated Pi uptake to the cytosol. That is, the PI3K/AKT pathway of receptors is responsible for insulin-regulated glucose uptake as well as Pi transports [20]. On the other hand, this pathway is also responsible for the development of obesity and T2D [21]. Activation of this pathway could alleviate obesity and insulin resistance, while the disturbed regulation of PI3K can cause many human diseases, e. g., obesity. It cannot be a coincidence.

It seems that all metabolic actions of insulin can be deduced from Pi transport. These are protein phosphorylation, cytoplasmic alkalinization, glucose uptake and transport, glycolysis, enhancement of ATP formation of mitochondrial oxidative phosphorylation. One possibility would be that insulin Pi transport would take place in the alkalotic ion form $\left(\mathrm{HPO}_{4}{ }^{2-}\right)$. In this way, the procedure of the alkalinization of the cytoplasm would have been solved at the same time. Currently, no known transporter delivers bivalent phosphate ions into all cell types. Among the known Pi transpor- ters, PiT1 would be the most obvious since it was shown to play a role in cell proliferation [22], which phenomenon also requires insulin action. However, the transport capacity of PiT1 is too small - according to Beck.

Insulin transports likely monovalent $\mathrm{H}_{2} \mathrm{PO}_{4}^{-}$because immediately after its action, $\mathrm{Na}^{+} / \mathrm{H}^{+}$exchanger (NHE) of the target cells start working [17]. However, there are high capacity $\mathrm{Na}^{+} / \mathrm{H}^{+}$ion-exchangers (pumping $\mathrm{H}^{+}$out of the cells several minutes to some hours), this intervallum fits insulin action. The increased cytosolic $\mathrm{Na}^{+}$ also can trigger $\mathrm{Na}^{+} / \mathrm{K}^{+}$-ATPase function [23]. Insulin itself promotes $\mathrm{Na}^{+} / \mathrm{K}^{+}$-ATPase indeed [24]. (After 2-3 hours, intracellular $\mathrm{K}^{+}$concentration and the membrane potentials will also grow.) All of these processes require ATP energy, so there is a great need for increased oxidative phosphorylation. If the NHE exchanger plays a central role in insulin action, it is easier to understand how can relatively mild but chronic cytosolic acidosis cause insulin resistance; exhausting the capacity of the NHE exchanger. The cytosolic Pi transport and alkalinization together are a strong signal (and also excipient) for mitochondrial ATP formation [16,25].

GH, IGF-I, and insulin all increase Pi reabsorption in the proximal tubules of the kidneys, thus causing a positive phosphate balance [26]. 1,25 dihydroxy-vitamin D3 also increases phosphate reabsorption. If the precondition for anabolic effect is a positive Pi balance, then vitamin D3 can have an anabolic effect, as was indicated by some data [27]. Activated vitamin D3 (calcitriol) functions as a hormone and stimulating the $\mathrm{Na}^{+} / \mathrm{Pi}$ cotransport [28]. The synergism of GH, IGF-I, and insulin is well known but, surprisingly, relationships could be found between insulin and vitamin D3 as well as between diabetes and vitamin D deficiency. Treating the lack of vitamin D with supplements did promote not only the improvement of osteoporosis but also reduced high blood sugar levels in diabetes and high blood pressure in hypertension [29]. (It is important to note here that we are talking about making up for a deficiency and not a pharmacological effect.) It was found that $70 \%$ of Type 2 Diabetic patients were suffering from hypovitaminosis D. Vitamin D, giving to children in the first year of life in a slightly higher dose (2000 IU/day), significantly reduced the occurrence of Type 1 Diabetes [30]. In summary: Insulin, IGF-I (also other Growth Factors), and the activated vitamin D3 have a common characteristic feature; they are capable of helping the transport of inorganic phosphate into their target cells. According to the author of this book, that is why they have an anabolic effect. 
Therapeutic attempts to eliminate insulin resistance - giving nutrients

Many have dealt with the role of magnesium deficiency in insulin resistance and supposed that the administration is a potent complementary agent to eliminate insulin resistance. There is a broad consensus that lack of $\mathrm{Mg}^{2+}$ would have a common additional harmful effect in Metabolic Syndrome-related disorders (insulin resistance, hypertension, obesity, dyslipidemia) [12,31]. According to Barbagallo, the $\mathrm{Ca}^{2+} / \mathrm{Mg}^{2+}$ antagonism plays a crucial role; the cytosolic $\mathrm{Ca}^{2+}$ increase would participate in postreceptorial insulin resistance. The most, but not all, trials confirmed the beneficial effect of magnesium administration in T2D [4]. A variety of magnesium salts was used for supplementation; the most surprising is the recently given $\mathrm{MgSO}_{4}$, which seems to be also useful in humans [32]. The effectivity of administering $\mathrm{MgSO}_{4}$ is unexpected because sulfate salts have very poor absorption. However, sulfate may also have a therapeutic effect as - according to Golden - sulfate is a Type II nutrient (see Part 1. [24]).

Physiologic and pathophysiologic effects of zinc are similar to magnesium in T2D. The beneficial effects of $\mathrm{Zn}^{2+}$ alone to insulin resistance is similarly controversial as $\mathrm{Mg}^{2+}$, although administration of $\mathrm{Zn}^{2+}$ plus $\mathrm{Mg}^{2+}$ plus multivitamin supplementation could decrease HgA1C significantly [33].

The administration of $\mathrm{HCO}_{3}{ }^{-}$could decrease insulin resistance and increase glucose tolerance in renal metabolic acidosis [34]. By contrast, administering of $\mathrm{HCO}_{3}{ }^{-}$with the therapeutic goal in T2D - supposed that T2D was caused by dietary origin metabolic acidosis - did not yield the expected result [35]. According to the author of this book, that is because 1)The increased serum bicarbonate level does not elevate $\mathrm{pHi}$; contrary, it will decrease $\mathrm{pHi}$, causing hypoventilation-hypercapnia. 2) The metabolic alkalosis (elevated $\mathrm{HCO}_{3}^{-}$level) increases the Pi excretion through kidneys and decreases serum Pi levels. By contrast, hyperventilation hypocapnia promotes the transport of Pi ions into cells, although it also reduces Se-P levels (see Part 1. [35].).

Therapeutic attempts to eliminate insulin resistance - giving phosphate nutrients

The pathophysiological role of phosphates is exceptionally controversial; most researchers and clinicians see only the risk, although it is unclear how would harm. Several articles address the pathophysiology of high serum Pi, particularly in cardiovascu- lar risk. Serum phosphate levels seem to be the determining factor of the harm; it would be a 'double-edged weapon' [36]. On the other hand, several papers demonstrated the causative association between low serum Pi, Metabolic Syndrome, and T2D [11,37,38]. Investigation of serum phosphate levels as a single pathogenic factor is overly mechanical, not least because Metabolic Syndrome is widely accepted as the leading player of the cardiovascular risk [12]. The issue can be clarified, knowing the exact pathophysiologic role of phosphates and the regulation disturbances of serum phosphate levels. Recent research suggests that increasing phosphate uptake enhances energy expenditure through fatty acid utilization [39] and inhibits fat deposition. It is hypothesized that increased Pi uptake into the cells increases ATP concentration, for example, in the muscular [26], hepatic [40], and hypothalamic cells (see Part 3. [49]), which suppresses appetite and hinder food uptake. However, the exact mechanism is not known.

Steen Jaedig and Nels Henningsen were ahead of their ages with their patent [41] and their research results $[42,43]$. The inventors investigated the acute effect of $\mathrm{K}^{+}(35-40 \mathrm{mmol}), \mathrm{Mg}^{2+}$ (17mmol), and dibasic $\mathrm{HPO}_{4}{ }^{2-}(35 \mathrm{mmol})$ salts on energy expenditure and found it to be significantly elevated. They logically deduced that the salt combination would work, and it worked. Their patent [41] declared that the proposed salt mixture has a beneficial effect on the slightly increased blood pressure, overweight and mild Type 2 Diabetes, too. The long-term impact of potassium-magnesium phosphate was filed as a patent. They achieved a steady weight loss of $8.6 \mathrm{~kg}$ on average. Oxygen consumption remained elevated over three months. What was the fate of the invention, what did it materialize? - we do not know it.

Omar Obeid and colleagues rediscovered the increased energy expenditure, in particular, the postprandial expenditure, as a consequence of adding various phosphate salts, and they filed a patent [44]. Many of their noteworthy papers have been published on the subject $[38,45]$. They presented a recent randomized clinical trial, and their final report is: "phosphorus supplementation for 12 weeks significantly decreases body weight, BMI, waist circumference and subjective appetite scores" [40].

Therapeutic application of insulin - in unusual ways or of nondiabetic patients

Insulin is also an anabolic ('anticatabolic') hormone most often used in clinical practice. It is used as a medicine in diabetes, and 
it is also applied to treat patients suffering from other illnesses. Insulin affects the accumulation of CBIs in the cytosol, increases ATP production, and anabolism. The method - was introduced first by the Mexican Sodi-Pallarés - has been subject to fierce debates since its beginning. The manner still has many adherents, and many publications have appeared for now. One of the methods of Sodi-Pallarés and his follower was that they administered the three metabolic-enhancement components (GIK=glucose + insulin + potassium) in infusion to patients having postmyocardial infarction or various organic heart diseases [46]. There are ample results, some of which seem to verify the beneficial effects of the GIK method but others not being so conclusive. It is well known that insulin, combined with glucose, can promote the transport of potassium into cells. This fact in itself can justify the applicability of insulin to nondiabetic patients. The anabolic effect of insulin can be useful in the case of several diseases. It was found that insulin is also valuable for the following nondiabetic disorders to increase anabolism, promote healing or stabilize critical illnesses: wound healing, parenteral nutrition, managing of septic shock, different drug-overdoses and other emergency diseases [47]. Briefly, insulin has a recovery effect - almost independently of the underlying condition.

The ability of insulin to increase the cytosolic potassium levels is not a primary action, but a result of several indirect effects (see above). It was proved (see Part 3. [3]) that transports of glucose and phosphate (in this way, the ATP synthesis) are actions of insulin, and they are damaged in the skeletal muscles in the case of insulin resistance. It is a logical conclusion that these damages form the biochemical basis of insulin resistance. The causes and consequences of insulin resistance are the decrease of Pi and the increase of $\mathrm{H}^{+}$concentration in cytosol, as well as hindered oxidative phosphorylation of glucose (see Part 3.). It was also declared that the defects are rooted in the same problem. Somehow, the insulin-mediated glucose and phosphate transports can explain the anabolic effects of insulin. The damaged phosphate transport establishes catabolism in insulin resistance.

Physiologically, insulin does not only provide efficient glucose transport to cells but also promotes ATP production by increasing the $\mathrm{HPO}_{4}{ }^{2-}$ concentration in cytosol, facilitating the operation of individual ion pumps, although its details are not known. This way, increased cytoplasmic $\mathrm{HPO}_{4}{ }^{2-}$ concentration - generating ATP production - enables the transport of cations (e.g., $\mathrm{K}^{+}, \mathrm{Mg}^{2+}$, and $\mathrm{Zn}^{2+}$ ) into the cells. We have to note here that a primer ion transport (like the transport of $\mathrm{Pi}$ in this particular case) initiates a cascade of secondary ionic carriers and metabolic changes that operate according to rules not fully understood.

Insulin resistance is both a cause and a consequence of the lack of insulin action. On the one hand, it is evidenced that intensive insulin therapy can reduce insulin resistance in Type 2 Diabetes, compared to oral antidiabetic treatment [48]. That is also a vicious circle in the pathophysiology of T2D. On the other hand, according to a Hungarian patent application, an RDA-proportioning salt-mixture of $\mathrm{HPO}_{4}{ }^{2-}, \mathrm{H}_{2} \mathrm{PO}_{4}{ }^{-}, \mathrm{K}^{+}, \mathrm{Mg}^{2+}$, and $\mathrm{Zn}^{2+}$ minerals were useful in experimental diabetes for the observed animals, both in reducing insulin resistance and decreasing in cardiovascular side effects of rosiglitazone [49]. The solution seems obvious: The two effective methods to reduce insulin resistance and increase anabolic effects should be combined since they form a unit. Insulin transports Pi into cells, followed by glucose, $\mathrm{K}^{+}, \mathrm{Mg}^{2+}$, and $\mathrm{Zn}^{2+}$ transports. It would be a modified Sodi-Pallarés therapy that could apply to both diabetes and other conditions (see above).

The fact that we can use insulin as a universal anabolic hormone in a catabolic state (almost independently of the underlying disease) also justifies the validity of the metabolic model created by the author of this book (see Part 1.). Insulin injects energy into the target cells in the form of glucose, and a second messenger in the form of $\mathrm{HPO}_{4}{ }^{2-}$. In this way, initiates an anabolic program: it generates ATP, supplies energy and phosphate for phosphorylation, enhances the active transport of the Cytoplasm Builder Ions to the cytosol, increases membrane potential, and eventually initiates the cytoplasmic protein building [50]. Previously, the author of this book predicted that a proper mixture of CBIs would not only be able to influence diabetes in the desired direction, but the ion-combination can also reduce the side effects of certain drugs. This thesis was later confirmed [49].

Therapeutic usage of Cytoplasm Builder Ions (CBIs) together - without insulin

According to the hypothesis of the author of this book - the primary cause of Metabolic Syndrome and T2D would be latent intracellular acidosis and related ion-alterations. The low-grade acidosis slowly alters metabolism, Metabolic Syndrome-related Disorders gradually evolve and can induce insulin resistance, obesity, hypertension, dyslipidemia, hyperuricemia, etc. According to 
Psychosomatic Molecular Mechanisms of Metabolic Syndrome and Type 2 Diabetes. Part 4. A Recovery Strategy from Chronic Hypercapnia and Metabolic Syndrome-Related Disorders, (a Research Proposal)

the hypothesis, metabolism is controlled by the Momentary Intracellular Ion-pattern (see Part 1.).

Recovery from acidosis, in the presence of insulin, is similar to refeeding syndrome (see Part 1. [31]), [45]. Cell metabolism recovering from low degree acidosis is starting to recover to original states, which requires the presence of anabolic pathways. Immediately after Pi uptake and alkalization of cytoplasm, are also ingested $\mathrm{K}^{+}, \mathrm{Mg}^{2+}$, and $\mathrm{Zn}^{2+}$ to the cells' cytoplasm, which is followed by the uptake of amino acids (positive $\mathrm{N}$ balance, see Part 1.). The increased levels of $\mathrm{HPO}_{4}^{2-}$ in the intracellular space appear to trigger anabolic response, the recovery. However, that state can also be achieved by a straightforward method. Administration of monobasic phosphate salts (e. g., $\mathrm{KH}_{2} \mathrm{PO}_{4}$ ) increases ventilation by its acidic effect, and the hypocapnia introduces phosphates into the cells through alkalizing cytosol. The increased tissular glycolysis can its explanation (see Part 1.[9]). And from that on, the runoff would be similar to what the insulin would trigger. By contrast, when bicarbonate was administered, the exact opposite will happen. Bicarbonate is an alkali, which triggers hypoventilation [35]. The elevating $\mathrm{pCO}_{2}$ level will decrease $\mathrm{pHi}$ in the cells, and intracellular acidosis will develop, and phosphates will be excreted into the urine (see Part 1. [35]).

The author of this book has tried monobasic phosphate therapy for himself three times. First-time (thirteen years ago) severe social-mental stress resulted in acute depression, hypertension, and ten kg gain in a short period (about one month). The treatments were based on logical considerations, as well as the observations of Jaedig and Henningsen $[41,42,43]$. With the addition of $2 \times 1 \mathrm{~g}$ $\mathrm{KH}_{2} \mathrm{PO}_{4}$ and $2 \mathrm{x} 2 \mathrm{~g}$ Magnesium citrate daily, the $10 \mathrm{~kg}$ body weight gain disappeared, tension and mood also improved about three weeks. (Fat had increased the abdominal circumference, which was vanished beside the salt treatment.) After that, the stress situation ceased, and the salt combination was abandoned; unfortunately, the weight gain of $10 \mathrm{~kg}$ also slowly returned, although depression and hypertension did not. Since then, he has not taken any antihypertensive or other drugs.

The second time, the author started taking $2 \mathrm{x} 0,8 \mathrm{~g} \mathrm{KH}_{2} \mathrm{PO}_{4}$ salt daily, in monotherapy, two years ago to reduce his body weight. After about one month of treatment, symptoms of tetany occurred while the body weight did not decrease. Blood tests showed significant hypocalcemia, hypokalemia, hypomagnesemia, and hypo- phosphatemia. Serum cholesterol and LDL-cholesterol decreased by $15 \%$ to the normal level; the other parameters did not change significantly.) After that, he suspended the salt-administration.

For the third time (nearly a year ago), the author started to take in $1 \mathrm{~g} \mathrm{KH}_{2} \mathrm{PO}_{4}$ daily with $2 \mathrm{~g} \mathrm{Mg}$-citrate and $0.3 \mathrm{~g} \mathrm{CaCl}_{2}$ in the morning. After 4 - 6 weeks of taking salts, without changing his lifestyle or diet, he began to lose weight. He lost $8 \mathrm{~kg}$ in the next four weeks, and then stopped losing weight - partly because he was careful and did not want to decrease more. The dose was reduced to 0,5 $\mathrm{g}$ of $\mathrm{KH}_{2} \mathrm{PO}_{4}$ and $1 \mathrm{~g} \mathrm{Mg}$-citrate (the $\mathrm{CaCl}_{2}$ was paused.). Then he holds his reduced bodyweight. His BMI is 30 currently. What has changed? After administering the salt combination, the feeling of hunger radically decreased, though the appetite came with meals, maybe even increased, and the sense of the satiety was delayed. Therefore, attention had to be paid to the volume of the meal. A part of the contradiction is that healing is a long process, and the other hand, the nutritional supplementation is not perfect yet. Thirdly, lifestyle, and behavior also play a role. Fitness has improved dramatically: both muscle strength, endurance, fatigue tolerance, etc. Despite this, he just walked as before (8,000 steps a day). Mental functions have also become much more vigorous, but there were sometimes irritability, impatience, and excitement. (These are the symptoms of the hyperarousal, which refers to sympathicotonia or adrenergic actions). Explanation: The intracellular alkalosis probably sometimes overshooting, which means increased ATP turnover too. It also means that the experiment is not safe if the subject has no energy reserves; for example, having coronary insufficiency, anything could happen. Therefore, self-testing is not recommended to anyone else, only under strictly controlled experimental conditions.

Initially, falling asleep was difficult due to increased arousal. Then the depth of sleep improved. According to the smart watch's sleep program, snoring fell from $8 \%$ to below $2 \%$ on average over three months. (Previously, a sleep therapist expert informally reported that similar salt combinations reduced the apnea-hypopnea index all ten observed patients with OSAS, after four weeks of treatment.) After five months of treatment, serum electrolytes $\left(\mathrm{K}^{+}, \mathrm{Mg}^{2+}\right.$, $\mathrm{Ca}^{2+}$, and Pi) were still at low-normal levels. Serum triglycerides decreased by $50 \%$, to the normal level, which has never been regular before for decades. LDL-cholesterol level in the normal range continued to decline; HDL-cholesterol did not change. After a six weeks break, lipid levels increased to high levels above the base- 
line. Bodyweight has not altered significantly after one month of the therapy pause. Combining therapy with traditionally proven methods (diet, exercise) would bring much better results.

Neither the Henningsen-Jaedig pair [41,42,43] nor the Obeid team [44] realized that the two physiological phosphate ions $\left(\mathrm{HPO}_{4}{ }^{2-}\right.$ and $\left.\mathrm{H}_{2} \mathrm{PO}_{4}{ }^{-}\right)$are regulated in entirely different ways by membrane transporters, hormones, and cytokines. They are different messengers for the cytoplasm, that is, their controls should be separate. At the same time, they can easily convert to each other due to changes in $\mathrm{pH}$. It means incredibly high flexibility and, at the same time, high sensitivity in the management of metabolism. For example, the two ions together are one of the essential intracellular buffers, which is a passive role. By contrast, the second messenger role of phosphates is one of the most active, similar to $\mathrm{Ca}^{2+}$. These dynamic, dialectical contradictions are complicated - sometimes impossible - to resolve. If the hypothesis will be confirmed that the proportion of dibasic and monobasic phosphate ions and the amount of Pi administered can control the volume of respiration, $\mathrm{pCO}_{2}$ level, and thereby the metabolism of cells in the whole body; this would be extremely surprising and frustrating discovery.

The metabolism of the body necessarily needs a significant degree of inertia, because, without it, it would not be able to have relative stability. If we want to control the respiration and thereby the metabolism of the body through the ratios of ingested phosphates, it is similar to if a smaller ship would be pulling a smaller iceberg towards a specific target, to which a larger one is attached. The little one could move relatively easily, but the big iceberg is almost motionless. It may give the impression that the ship cannot move them. In fact, the big one is also moving slowly in the desired direction. The small iceberg symbolizes the breathing, while the big ice the metabolism of the body, and the ship symbolizes the ions; the two phosphate ions relation to each other are controlling the rate of motion. It may be valid that the intake of phosphates restores the postprandial energy expenditure $[43,44]$ immediately - in the long run, they can also control the rate of the metabolism of the body. Metabolic Syndrome develops over the years, decades, so it can be expected that it will take months (maybe years?) to restore metabolism, if at all, would be reversible. In this way, prevention seems much more comfortable.

However, breathing change is only the first step; intracellular alkaline $\mathrm{pH}$ automatically increases metabolism when $\mathrm{pCO}_{2}$ decreases. Initially, the increase in ATP production capacity outweighs its consumption, i. e. restores the pathological balance (assuming previous chronic hypercapnia). It is a crucial fact that decreasing
$\mathrm{pCO}_{2}$ introduces the phosphate into the cytoplasm, thus retaining it, resulting in a positive phosphate balance in the body (see Part 1. [35]). The decreased serum phosphate, the hypophosphatemia can be dangerous. Phosphate transport to the inside of the cytoplasm and rising $\mathrm{pHi}$ is the constellation that insulin also generates, and it automatically initiates cytoplasm production, namely the anabolism. Pi uptake in cells is followed by $\mathrm{K}^{+}, \mathrm{Mg}^{2+}, \mathrm{Zn}^{2+}$, and other 'cytoplasm builders,' the Type II nutrients (e. g., amino acids) (see Part 1. [24]); the anabolism begins. The significant increase in cytoplasm mass (BCM) appears to last for months. That may represent a very substantial cytoplasm deficiency already in the early stages of Metabolic Syndrome, even much more in T2D. The positive $\mathrm{Ca}^{2+}$ balance of bones (low Se-Ca, decreased urine excretion) - during recovery - may indicate a significant $\mathrm{Ca}^{2+}$ and Pi deficiency, which is 'hungry bone' syndrome (see Part 3, [40]). Proper instrumentation is required to perform blood, and balance tests and awareness is required to evaluate clinical and laboratory data for metabolism.

The new method, if it would be proven, could be used to improve all types of chronic hypercapnia (COPD, OSA, OSAS, OHD), as well as to treat Metabolic Syndrome-related Disorders. By contrast, a salt mixture containing $\mathrm{H}_{2} \mathrm{PO}_{4}^{-} / \mathrm{HPO}_{4}^{2-}$ buffer in other proportions may also be suitable for treating of hyperarousal psychiatric disorders (anxiety, etc.) and cases of emergency hypocapnia disorders. The modified Sodi-Pallarés method therapy is expected to work if the intracellular metabolic acidosis dominates the catabolic state while the other in the case of respiratory acidosis would dominate.

\section{Conclusion}

The insulin signaling system is one of the most sophisticated systems known today. In contrast, insulin receptors appear already in primitive beings, confirming that insulin action plays an indispensable role in basic physiological processes. The effects of insulin enable growth-developmental and other life-events resulting from anabolism (see Part 1. and Part 2.). In Part 1., the author introduced the growth spiral of cytoplasm (CBIs - ATP - proteins), which is triggered by 'something.' Life has to be protected against the 'equalization effect' of The Second Law of Thermodynamics. That 'something' that starts and keeps moving the virtuous circle of 'growth-spiral' is mainly the insulin second messenger and signaling system. As parents, we do not need to know how the fetus develops, as physicians, we do not need to understand how the complex membrane receptors and signaling pathways of insulin function. Insulin can act without our consciousness if the all essential cytoplasm builder materials are available in the wanted proportions - we physicians 
are only auxiliary workers who serve the healing.

According to the hypothesis, the anabolic growth spiral is triggered by insulin delivering $\mathrm{HPO}_{4}^{2-}$ to the cytosol of the target cells. It is not known how many steps does it that and exactly how. The next moves are already recognized (see Part 1). The author suggests two simple methods (tricks) to mimic the second messenger signaling of insulin to deliver $\mathrm{HPO}_{4}{ }^{2-}$ into the cytosol of malfunctioning cells that are still reparable. Just as the cytosolic $\mathrm{pH}(\mathrm{pHi})$ determines the ratio of phosphates $\left(\mathrm{HPO}_{4}{ }^{2-} / \mathrm{H}_{2} \mathrm{PO}_{4}{ }^{-}\right)$to each other, that is also the case vice versa: the ratio of the two phosphate ions determines the pHi of the cytoplasm. Likewise, it is a crucial fact that the variable (decreasing or increasing) $\mathrm{pCO}_{2}$ changes the pHi immediately. That is, if we stimulate breathing by increasing its volume, we increase the pHi and decrease the intracellular $\mathrm{H}^{+}$ concentration. Due to the increasing pHi, cytosol uptakes the $\mathrm{Pi}$ ions from the extracellular space (presumably due to increased metabolism by alkaline media, which requires Pi ions because of increased ATP production). And as the $\mathrm{pH}$ of the cytosolic becomes alkaline, the ratio of $\mathrm{HPO}_{4}{ }^{2-} / \mathrm{H}_{2} \mathrm{PO}_{4}{ }^{-}$increases. If the 'cytoplasm builder' nutrients, ions are available in the extracellular space, and sufficient amount of oxygen also available, the growth of metabolism is not hindered, but it will be even accelerated; an anabolic metabolism, a virtuous circle, occurs. If, on the other hand, the patient is suffering from severe irreversible damages and his or her organs are unable to accelerate metabolism because, for example, arterial cerebral or cardiac coronary artery stenosis, the hypocapnia can be life-threatening.

The recovery strategy is based on the assumption that intracellularly acidosis prevails, which causes negative phosphate balance and catabolism in the Metabolic Syndrome and its related disorders, e. g., Type 2 Diabetes. The author proposes two recovery strategies.

1. The modified Sodi-Pallarés therapy is recommended to use in the cases of Metabolic Syndrome and other chronic organic disorders in the form of infusion. For the reasons described in Part 1., the most chronic organ diseases have intracellular metabolic acidosis, first of all, for energetical reasons. The deficiency of cytoplasm usually also presents in most of the chronic metabolic disorders. The infusion should contain phosphate buffer, potassium, magnesium, and zinc salts in addition to glucose and crystalline insulin. The proportions should be such that hypoglycemia does not develop.
2. The author would try the other method suggested by himself in patients with obesity or T2D, primarily with chronic lowgrade hypercapnia, if he had the possibility. It should be used monobasic, acidic $\left(\mathrm{H}_{2} \mathrm{PO}_{4}^{-}\right)$phosphate of $\mathrm{K}^{+}$and $\mathrm{Mg}^{2+}$ salts. The recommended doses of phosphate can attain RDA (Recommended Dietary Allowance) (300-700 mg/die phosphate) until the desired effect was achieved. The target is low grade, persistent hypocapnia $\left(\sim \mathrm{pCO}_{2} \leq 35 \mathrm{mmHg}\right)$. After reaching the target, let's reduce the dose of $\mathrm{H}_{2} \mathrm{PO}_{4}-$ or add a defined amount of acidic phosphate buffer $\left(\mathrm{H}_{2} \mathrm{PO}_{4}{ }^{-} / \mathrm{HPO}_{4}{ }^{2-}\right)$ in the form of $\mathrm{K}^{+}$, $\mathrm{Mg}^{2+}$, and $\mathrm{Zn}^{2+}$ salts instead. Blood tests and clinical pictures determine the salt composition and dosages be administered. Hypocapnia, following chronic hypercapnic acidosis, can also provoke hypocalcemia and hypophosphatemia due to 'hungry bone syndrome' (see Part 1.). Both 'hungry bones' and 'hungry tissues' can last for months. It can take many months while we make up for missing cytoplasmic and electrolyte deficiencies. In the clinical practice, that results in positive $\mathrm{K}^{+}, \mathrm{Mg}^{2+}$, $\mathrm{Zn}^{2+}, \mathrm{Pi}$, and $\mathrm{N}$ balances. Hypophosphatemia, hypokalemia, and hypocalcemia also often occur. We can avoid severe serum electrolyte deficiencies with frequent blood tests. In addition to blood tests, electrolyte balance observations are also advised. The continuous monitoring of exhaled air (PETCO2) can be useful. Blood gas testing is important for accurate assessment and monitoring of respiratory disorders - let us follow the Base Excess! Once the desired goal has been achieved, it is recommended to use a custom-formulated phosphate-salt mixture to prevent relapse.

It is recommended to consider other known (and not yet known) 'cytoplasm builder' (Type II) nutrients (amino acids, unsaturated fats, trace elements, vitamins, etc.) when expanding the research (see Part 1. [24]). The tests should only be performed under medical control and according to the rules of research regulations. Self-experiment is dangerous and forbidden! We have to dispel the misconception that intracellular electrolytes can be replaced separately: CBIs can only be supplemented simultaneously by changing the intracellular $\mathrm{pH}$ into the desired direction. Then protein synthesis also occurs (see Part 1.). The demand for phosphates is hugely variable in different conditions, depending on whether the metabolic spiral is spinning into an anabolic or catabolic direction. In the former case, with proper renal function, high doses can be given. It seems that the phosphate is not toxic, but the hyperphosphatemia is dangerous. (The main reason for toxicity is that phosphate solubility is small.) Phosphate toxicity is particularly low when breathing is increased as Pi migrates into cells, and

Citation: András Sikter. "Psychosomatic Molecular Mechanisms of Metabolic Syndrome and Type 2 Diabetes. Part 4. A Recovery Strategy from Chronic Hypercapnia and Metabolic Syndrome-Related Disorders, (a Research Proposal)". Acta Scientific Medical Sciences 4.3 (2020): 150-161.

DOI: 10.31080/ASMS.2020.04.0578

Published: February 29, 2020 
Psychosomatic Molecular Mechanisms of Metabolic Syndrome and Type 2 Diabetes. Part 4. A Recovery Strategy from Chronic Hypercapnia and Metabolic Syndrome-Related Disorders, (a Research Proposal)

serum phosphate decreases to the direction of hypophosphatemia. In contrast, when breathing decreases, Se-Pi levels increase, and urinary excretion of phosphate also raises. Negative Pi balance may be explained by intracellular acidosis and catabolism.

It is not yet known how far Metabolic Syndrome-related disorders are reversible, although obesity is revocable. Proven methods (diet, exercise) increase the effectiveness of the therapy. In summary: insulin can achieve recovery by triggering the cytoplasmic growth-spiral. That is, the 'primum movens' of cytoplasmic growth is the increase of cytosolic $\mathrm{HPO}_{4}{ }^{2-}$ concentrácion as the second messenger of insulin. It seems we have other methods to attain that.

\section{Bibliography}

1. Guerrero-Romero F and Rodríguez-Morán M. "Low serum magnesium levels and metabolic syndrome". Acta Diabetologica 39.4 (2002): 209-213.

2. Kao WH., et al. "Serum and dietary magnesium and the risk for type 2 diabetes mellitus: the Atherosclerosis Risk in Communities Study". Archives of Internal Medicine 159.18 (1999): $2151-2159$.

3. Simmons D., et al. "Hypomagnesaemia is associated with diabetes: Not pre-diabetes, obesity or the metabolic syndrome". Diabetes Research and Clinical Practice 87.2 (2010): 261-266.

4. Barbagallo M and Dominguez LJ. "Magnesium and type 2 diabetes". World Journal of Diabetes 6.10 (2015): 1152-1157.

5. Chatterjee R., et al. "Potassium and risk of Type 2 diabetes". Expert Review of Endocrinology and Metabolism 6.5 (2011): 665-672.

6. Rowe JW., et al. "Effect of experimental potassium deficiency on glucose and insulin metabolism". Metabolism: Clinical and Experimental 29.6 (1980): 498-502.

7. Honnorat J., et al., "Effects of diabetes type and treatment on zinc status in diabetes mellitus". Biological Trace Element Research 32 (1992): 311-316.

8. Al-Maroof RA and Al-Sharbatti S. "Serum zinc levels in diabetic patients and effect of zinc supplementation on glycemic control of type 2 diabetics". Saudi Medical Journal 27.3 (2006): 344-350.

9. Singh U. "Zinc in relation to type 1 and type 2 diabetes: An overview". Journal of Applied and Natural Sciece 6.2 (2014): 898-903.
10. Cruz KJC., et al. "Zinc and insulin resistance: Biochemical and molecular aspects". Biological Trace Element Research 186.2 (2018): 407-412.

11. Håglin L. "Hypophosphataemia: cause of the disturbed metabolism in the metabolic syndrome". Medical Hypotheses 56.6 (2001): 657-663.

12. Kalaitzidis R., et al. "Disturbances of phosphate metabolism: another feature of metabolic syndrome". American Journal of Kidney Diseases 45.5 (2005): 851-458.

13. DeFronzo RA and Lang R. "Hypophosphatemia and glucose intolerance: evidence for tissue insensitivity to insulin". The New England Journal of Medicine 303.22 (1980): 1259-1263.

14. Zhou XJ., et al. "Phosphate depletion impairs insulin secretion by pancreatic islets". Kidney International 39.1 (1991): 120 128.

15. Lu Y., et al. "Serum amino acids in association with prevalent and incident Type 2 Diabetes in a Chinese population". Metabolites 9.1 (2019): E14.

16. Bose S., et al. "Metabolic network control of oxidative phosphorylation: multiple roles of inorganic phosphate". The Journal of Biologica Chemistry 278.40 (2003): 3955-3965.

17. Yang J., et al. "Insulin-stimulated cytosol alkalinization facilitates optimal activation of glucose transport in cardiomyocytes". American Journal of Physiology. Endocrinology and Metabolism 283.6 (2002): E1299-E1307.

18. Lim El., et al. "Measuring the acute effect of insulin infusion on ATP turnover rate in human skeletal muscle using phosphorus-31 magnetic resonance saturation transfer spectroscopy". NMR in Biomedicine 23.8 (2010): 952-957.

19. Klip A., et al. "Insulin-induced cytoplasmic alkalinization and glucose transport in muscle cells". The American Journal of Physiology 250.5 Pt1 (1986): C720-C728.

20. Pesta DH., et al. "Hypophosphatemia promotes lower rates of muscle ATP synthesis". FASEB Journal 30.10 (2016): 33783387.

21. Huang X., et al. "The PI3K/AKT pathway in obesity and type 2 diabetes". International Journal of Biological Sciences 14.11 (2018): 1483-1496. 
22. Beck L., et al. "Identification of a novel function of PiT1 critical for cell proliferation and independent of its phosphate transport activity". The Journal of Biological Chemistry 284.45 (2009): 31363-31374.

23. DeFronzo RA., et al. "Effect of graded doses of insulin on splanchnic and peripheral potassium metabolism in man". The American Journal of Physiology 238.5 (1980): E421-E427.

24. Sweeney G and Klip A. "Regulation of the Na+/K+-ATPase by insulin: why and how?" Molecular and Cellular Biochemistry 182.1-2 (1998): 121-133.

25. Taylor DJ., et al. "Effect of insulin on intracellular pH and phosphate metabolism in human skeletal muscle In vivo". Clinical Science (London) 81.1 (1991): 123-128.

26. Tenenhouse HS. "Regulation of phosphorus homeostasis by the type iia na/phosphate cotransporter". Annual Review of Nutrition 25 (2005): 197-214.

27. Brown TA. "Vitamin D analogs as anabolic agents". Journal of Musculoskeletal and Neuronal Interactions 6.4 (2006): 341343.

28. Katai K., et al. "Regulation of intestinal Na+-dependent phosphate co-transporters by a low-phosphate diet and 1,25-dihydroxyvitamin D3". The Biochemical Journal 343.Pt3 (1999): 705-712.

29. Zittermann A. "Vitamin D in preventive medicine: are we ignoring the evidence?" The British Journal of Nutrition 89.5 (2003): 552-572.

30. Harris SS. "Vitamin D in type 1 diabetes prevention". The Journal of Nutrition 135.2 (2005): 323-325.

31. Barbagallo M., et al. "Diabetes mellitus, hypertension and ageing: the ionic hypothesis of ageing and cardiovascularmetabolic diseases". Diabetes and Metabolism 23.4 (1997): 281-294.

32. Solati M., et al. "Oral magnesium supplementation in type II diabetic patients". Medical Journal of the Islamic Republic of Iran 28 (2004): 67.

33. Gunasekara P., et al. "Effects of zinc and multimineral vitamin supplementation on glycemic and lipid control in adult diabetes". Diabetes, Metabolic Syndrome and Obesity: Targets and Therapy 4 (2011): 53-60.
34. Kraut JA and Madias NE. "Consequences and therapy of the metabolic acidosis of chronic kidney disease". Pediatric Nephrology 26.1 (2011): 19-28.

35. Harris SS and Dawson-Hughes B. "No effect of bicarbonate treatment on insulin sensitivity and glucose control in nondiabetic older adults". Endocrine 38.2 (2010): 221-226.

36. Park W., et al. "Serum phosphate levels and the risk of cardiovascular disease and metabolic syndrome: a double-edged sword". Diabetes Research and Clinical Practice 83.1 (2009): 119-125.

37. Ditzel J and Lervang HH. "Disturbance of inorganic phosphate metabolism in diabetes mellitus: its impact on the development of diabetic late complications". Current Diabetes Reviews 6.5 (2010): 323-333.

38. Obeid OA. "Low phosphorus status might contribute to the onset of obesity". Obesity Reviews 14.8 (2013): 659-664.

39. Chun S., et al. "A High Phosphorus Diet Affects Lipid Metabolism in Rat Liver: A DNA Microarray Analysis". PLoS One 11.5 (2016): e0155386.

40. Ayoub JJ., et al. "Effect of phosphorus supplementation on weight gain and waist circumference of overweight/obese adults: a randomized clinical trial". Nutrition and Diabetes 5 (2015): e189.

41. Henningsen $\mathrm{N}$ and Jaedig S. "Composition to improve cellular metabolism". (1986).

42. Jaedig $S$ and Henningsen NC. "Increased metabolic rate in obese women after ingestion of potassium, magnesium- and phosphate-enriched orange juice or injection of ephedrine". International Journal of Obesity 15.6 (1991): 429-436.

43. Jaedig S., et al. "Increased postprandial energy expenditure in obese women after peroral K- and Mg-phosphate". Mineral Electrolyte and Metabolism 20.3 (1994): 147-152.

44. Obeid 0., et al. "Composition and use of macro-mineral glycemic response and reduce body weight". U. S (2017).

45. Obeid OA., et al. "Refeeding and metabolic syndromes: two sides of the same coin". Nutrition and Diabetes 4 (2014): e120.

46. Allison SP., et al. "Insulin, glucose, and potassium in the treatment of congestive heart failure". British Medical Journal 3.5828 (1972): 675-678. 
Psychosomatic Molecular Mechanisms of Metabolic Syndrome and Type 2 Diabetes. Part 4. A Recovery Strategy from Chronic Hypercapnia and Metabolic Syndrome-Related Disorders, (a Research Proposal)

47. Benni JM and Patil PA. "Non-diabetic clinical applications of insulin". Journal of Basic and Clinical Physiology and Pharmacology 27.5 (2016): 445-456.

48. Wang D., et al. "Effects of intensive insulin therapy upon pancreatic $\beta$ cell function in patients newly diagnosed with type II diabetes". International Journal of Clinical and Experimental Medicine 8.1 (2015): 1391-1395.

49. Szilvássy Z and Peitl B. "Gyógyszerösszetétel inzulin érzékenyítő hatás fokozására Composition of medicine for enhancing insulin sensitization". Hungarian Patent Application (2013).

50. Sakurai Y., et al. "Stimulation of muscle protein synthesis by long-term insulin infusion in severely burned patients". Annals of Surgery 222.3 (1995): 283-297.

\section{Assets from publication with us}

- Prompt Acknowledgement after receiving the article

- Thorough Double blinded peer review

- Rapid Publication

- Issue of Publication Certificate

- High visibility of your Published work

Website: https://www.actascientific.com/

Submit Article: https://www.actascientific.com/submission.php

Email us: editor@actascientific.com

Contact us: +919182824667

Citation: András Sikter. "Psychosomatic Molecular Mechanisms of Metabolic Syndrome and Type 2 Diabetes. Part 4. A Recovery Strategy from Chronic Hypercapnia and Metabolic Syndrome-Related Disorders, (a Research Proposal)". Acta Scientific Medical Sciences 4.3 (2020): $150-161$.

DOI: 10.31080 /ASMS.2020.04.0578

Published: February 29, 2020 